\title{
Dinámica espacio-temporal del contenido en nitrato de las aguas su- perficiales y subterráneas de la cuenca del río Oja (La Rioja, España): Vulnerabilidad del acuífero aluvial
}

\author{
Mercedes Arauzo ${ }^{1, *}$, María Valladolid ${ }^{2}$, Juan José Martínez-Bastida ${ }^{1}$ y Carmen Gutiérrez ${ }^{3}$ \\ 1 Departamento de Contaminación Ambiental. Centro de Ciencias Medioambientales-CSIC. Serrano 115 dpdo. \\ 28006 Madrid. \\ 2 Departamento de Biodiversidad y Biología Evolutiva. Museo Nacional de Ciencias Naturales-CSIC. José Gu- \\ tiérrez Abascal 2. 28006 Madrid. \\ ${ }^{3}$ Departamento de Agroecología. Centro de Ciencias Medioambientales-CSIC. Serrano 115 dpdo. 28006 Madrid.
}

* Autor responsable de la correspondencia: mercedes@ccma.csic.es

\begin{abstract}
RESUMEN
Dinámica espacio-temporal del contenido en nitrato de las aguas superficiales y subterráneas de la cuenca del río Oja (La Rioja, España): Vulnerabilidad del acuífero aluvial

La escorrentía y filtración de aguas con alto contenido en nitrato procedente de las prácticas de fertilización agrícola, genera un grave problema de contaminación difusa de los recursos hídricos. Como resultado, se alcanzan concentraciones de nitrato en las reservas de agua que pueden afectar a la salud humana y a la calidad ambiental. En Europa la norma encargada de regular esta problemática es la Directiva 91/676/CEE, en la que se define como zona vulnerable aquella superficie territorial cuya escorrentía o filtración pueda originar contaminación de las aguas por nitratos. La Directiva fija en $50 \mathrm{mg} / \mathrm{l}$ de nitrato el límite a partir del cual se entiende que el agua está afectada por el proceso de contaminación.

Con frecuencia estacional y durante un periodo superior a un ciclo anual, se ha realizado un seguimiento de la evolución piezométrica del acuífero aluvial del Oja y una caracterización hidroquímica del mismo y del río Oja (La Rioja, España). Parte del área de estudio está tipificada por la Comunidad de La Rioja como zona vulnerable y zona vulnerable ampliable. Nuestro objetivo ha sido estudiar la dinámica del contenido en nitrato de las aguas superficiales y subterráneas de la Unidad Hidrogeológica del aluvial del Oja, mediante la interpretación de una secuencia de mapas que ilustran las variaciones, en el espacio y en el tiempo, del proceso de contaminación. Se discute, además, la relación entre la distribución del ión nitrato y las variaciones hidrológicas, las características del sustrato geológico y el periodo de mayor aporte de fertilizantes.

Durante la estación estival, se observó un amplio descenso en el nivel piezométrico del acuífero, como consecuencia de las extracciones de agua por bombeo para riego agrícola y del menor aporte desde los arroyos tributarios. El agua también desapareció temporalmente de la zona media del cauce del Oja durante el verano. El caudal habitual se recuperó durante el otoño, con la subida del nivel piezométrico. La concentración de nitrato sobrepasó el nivel de referencia establecido en la Directiva en la mitad norte del acuífero aluvial. La lixiviación procedente del abono nitrogenado de primavera provocó una expansión del área contaminada hacia la zona centro-occidental del acuífero durante el verano, que remitió con la recarga de otoño-primavera. No ocurrió lo mismo en las zonas norte y este, con menor renovación del agua, donde las concentraciones de nitrato se situaron siempre por encima de los valores permitidos.
\end{abstract}

Palabras clave: Directiva 91/676/CEE, zonas vulnerables, nitrato, acuífero aluvial.

\section{ABSTRACT \\ Spatial and temporal dynamics of nitrate content in surface and grownwater of the Oja River catchment (La Rioja, Spain): Vulnerability of the alluvial aquifer}

A high content of nitrate in runoff and leaching water from excessive use of nitrogen fertilizer in farming generates a serious problem of diffuse pollution in water resources. As a result, hig nitrate concentrations in reservoir waters are reached which could produce harmful effects on human health and environment quality. In Europe, the standard that regulates this issue is the Directive 91/676/EEC that defines a vulnerable zone as an area of land that drains into waters, and that contains more than $50 \mathrm{mg} / \mathrm{l}$ nitrates, which is the limit stated by the Directive.

Once a season and during a period greater than an annual cycle, a hydrochemistry and piezometry study was performed in 
the Oja alluvial aquifer and the Oja River (La Rioja, Spain). A part of the study area was classified by Comunidad de La Rioja as a vulnerable zone and an expandable vulnerable zone. Our objective was to study the spatial and temporal dynamics of nitrate content in surface and subterranean waters of the basin River Oja, through the interpretation of a map sequence showing the temporal and spatial variation of the pollution processes. Relationships between nitrate ion distribution and period of fertilization, hydrological dynamics and geologic characteristics are discussed.

A marked fall in the aquifer piezometric level was observed during summer because of water extraction for agriculture and a lower water flow from the tributary streams. Water also disappeared in the middle zone of the River Oja during summer. Flow and the initial piezometric layer were recovered during next autumn. Nitrate concentration was higher than the reference level established by the Directive in the northern half of the alluvial aquifer. Nitrate leaching from fertilizers used in spring produced an expansion of pollution to the middle-west area of the aquifer during summer, which receded with the autumnspring recharge. Nitrate pollution in the northeast area of the aquifer did not show the same pattern, and always showed concentrations higher than the allowed level.

Keywords: Directive 91/676/EEC, vulnerable zones, nitrate, alluvial aquifer.

\section{INTRODUCCIÓN}

La infiltración y escorrentía de aguas con alto contenido en nitrato, derivada del efecto combinado de una excesiva fertilización nitrogenada y unas prácticas de riego poco optimizadas en las zonas agrícolas, contribuye al deterioro de los recursos hídricos. La lixiviación de ión nitrato desde la zona no saturada hacia los acuíferos no sólo se produce durante los periodos de cultivo, también puede desencadenarse durante las fases de intercultivo, por efecto de las lluvias (Arauzo et al., 2003). Como resultado, cada vez con más frecuencia, se alcanzan concentraciones que pueden afectar a la salud humana (contaminación de aguas de abastecimiento) y a la calidad ambiental (contaminación de acuíferos, procesos de eutrofización en ecosistemas acuáticos).

La contaminación del agua por nitratos afecta a todos los países de la Unión Europea (Nixon et al., 2000). La norma encargada de regular esta problemática es la Directiva 91/676/CEE (traspuesta por el Real Decreto 261/1996), cuyo doble objetivo es la reducción de la contaminación por nitratos ya existente y la prevención de la aparición de nuevos procesos contaminantes. En la Directiva se define como zona vulnerable aquella superficie territorial cuya escorrentía o filtración afecte, o pueda afectar, a la contaminación de las aguas por nitratos. Se consideran aguas contaminadas aquellas con contenido en nitrato superior a $50 \mathrm{mg} / \mathrm{l}$, así como las que manifiestan una ten- dencia creciente que haga prever la necesidad de medidas de protección. La Directiva obliga a los países miembros a identificar, cada cuatro años, las zonas vulnerables a la contaminación por nitratos que, en el caso de aguas subterráneas, corresponde a las áreas de recarga de los acuíferos afectados o en riesgo de estarlo. El incumplimiento de las medidas restrictivas obligatorias en las zonas vulnerables conlleva sanciones tales como la retirada de subvenciones para el sector agrícola en las zonas afectadas. La aplicación de la Directiva, aparte de sus beneficios medioambientales obvios, puede repercutir negativamente a corto-medio plazo sobre la economía y la gestión de importantes áreas de regadío. Por ello se hace indispensable el desarrollo de investigaciones encaminadas a la detección, caracterización y protección de las zonas vulnerables (Instituto Tecnológico Geominero de España, 1998; Ministerio de Medio Ambiente, 2001; Gobierno de La Rioja \& ECCYSA, 2003), al desarrollo de métodos para la elaboración de balances hídricos y de nitratos ajustados en la zona no saturada (Ramos \& Küche, 1999; Paltineanu \& Starr,1997; Starr \& Paltineanu, 1998; Fares \& Alva, 2000; Arauzo et $a l ., 2003)$ y a la mejora en las estrategias de riego y abonado en los agrosistemas (Archer \& Thompson, 1993; Román et al., 1996; Sánchez et al., 1998; Rass et al., 1999; Díez, 2000; Díez et al., 2000; Sánchez-Pérez et al., 2003; Vázquez et al., 2003; Ortúzar et al., 2003).

En la Unidad Hidrogeológica del aluvial del 


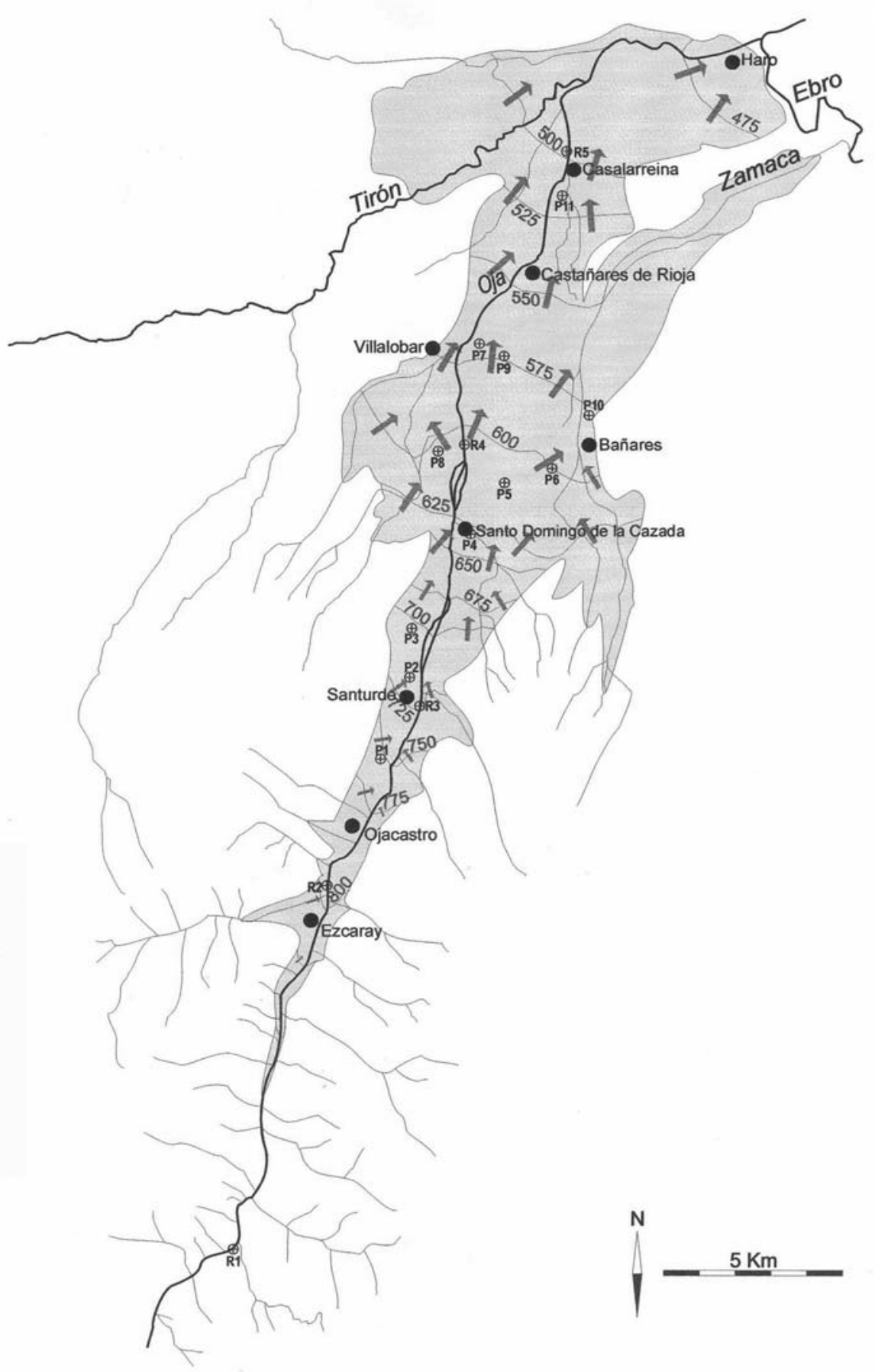

Figura 1. Situación de las estaciones de muestreo sobre el río Oja (R1-R5) y sobre la Unidad Hidrogeológica del aluvial del Oja (P1-P11). En color gris: Unidad hidrogeológica, donde se dibuja el mapa piezométrico (mayo de 2003) y el sentido del flujo del agua. Location of the sampling stations in the Oja River (R1-R5) and the Hydrogeological Area of the Oja alluvial (P1-P11). Hydrogeological area (in grey), with the piezometric map (May 2003) and the flow direction of the water. 
Oja (La Rioja Alta, España) encontramos una zona tipificada como vulnerable por la Comunidad de La Rioja (aluviales y terrazas del tramo bajo del Zamaca) y otra declara zona vulnerable ampliable (margen derecha del último tramo del acuífero aluvial del Oja). El proceso de contaminación surge de la interacción entre dos factores: la alta permeabilidad de sustrato geológico, hecho que favorece cualquier proceso de drenaje y lixiviación desde la zona no saturada hacia el acuífero subyacente, y la actividad económica dominante en la zona, basada en la agricultura intensiva de regadío. En este trabajo se ha realizado una caracterización química de las aguas superficiales y subterráneas de la Unidad Hidrogeológica del aluvial del Oja, centrando el objetivo principal de la investigación en el estudio de la dinámica espacio-temporal del contenido en nitrato en el río y en el acuífero aluvial. Se ha explorado la relación entre el proceso de contaminación y otros factores ambientales: aporte de fertilizantes, variaciones en el régimen hidrológico, sustrato geológico, etc. Se presentan los resultados relativos a quince meses de trabajo, con muestreos a escala estacional, si bien el equipo investigador se propone no interrumpir las campañas de muestreo, a fin de obtener información sobre las tendencias espaciotemporales del contaminante a largo plazo y, posteriormente, observar los efectos de la próxima aplicación de medidas restrictivas en el uso de fertilizantes sobre la calidad del agua en el conjunto río-acuífero.

\section{MATERIAL Y MÉTODOS}

El área de estudio se emplaza en la Unidad Hidrogeológica 09.04.03, también denominada aluvial del Oja, al norte de la Sierra de la Demanda (La Rioja, España). Coincide con la cuenca hidrográfica del río Oja, aunque también incluye el tramo final del río Tirón y gran parte del arroyo Zamaca. La principal actividad económica que se desarrolla en la zona es la agricultura intensiva (remolacha, patata, guisante, judía verde, cereal, frutales, viñedo), mayoritariamente en regadío. El régimen hidrológico es de tipo pluvionival, con una precipitación anual de $410 \mathrm{~mm}$ durante 2003 (estación meteorológica de Haro, página web del Gobierno de La Rioja). Los límites del acuífero aluvial del río Oja están definidos por la propia extensión de los aluviales, que ocupan una superficie de unos $148.5 \mathrm{~km}^{2}$. El espesor medio del acuífero es de $10 \mathrm{~m}$ (Instituto Geológico y Minero de España, 1985a). El sustrato geológico lo conforman depósitos del Cuaternario (coluviales, aluviales, terrazas aluviales, terrazas bajas y conos de pie de monte), con una litología de gravas gruesas aluviales, gravas poligénicas, arenas y limos (Instituto Geológico y Minero de España, 1985b). Se trata de un sustrato altamente permeable, con el nivel freático a escasa profundidad, hechos que favorecen los procesos de drenaje y lixiviación desde la zona no saturada del suelo y potencian la vulnerabilidad de las aguas subterráneas.

Desde mayo de 2003 a mayo de 2004 se ha realizado una caracterización hidroquímica del río Oja y de su acuífero aluvial. Las campañas de muestreo se han desarrollado con frecuencia estacional, en mayo, agosto y noviembre de 2003 y febrero y mayo de 2004. En la figura 1 se muestra la situación de las estaciones de muestreo sobre el río (R1-R5, desde cabecera hasta su confluencia con el río Tirón) y sobre el acuífero aluvial (P1-P11). En cada estación de muestreo (tanto en el río como en los pozos) se realizaron medidas in situ de temperatura del agua, $\mathrm{pH}$ y conductividad eléctrica. Para ello se utilizó un sistema multiparamétrico portátil conectado a multisonda y kit pH/re-dox, modelo YSI 556, marca YSI. Las medidas de coordenadas UTM y altitud se realizaron mediante un GPS modelo Garmin GPS 12, marca Garmin. En cada estación fluvial el caudal se estimó como el producto del área de la sección transversal del río y la velocidad de la corriente. Para mayor precisión, la sección transversal se dividió en subáreas, en las que se medía la velocidad de la corriente mediante un molinete Owens River Hydroprop. Posteriormente se calculó el caudal de cada subárea y se sumaron todos los caudales de las mismas para obtener, finalmente, el caudal de la sección transversal del río. En las estaciones P1-P11 se realizaron mediadas directas de la profundidad a la que se situaba el nivel freático (con relación a la superficie del sue- 


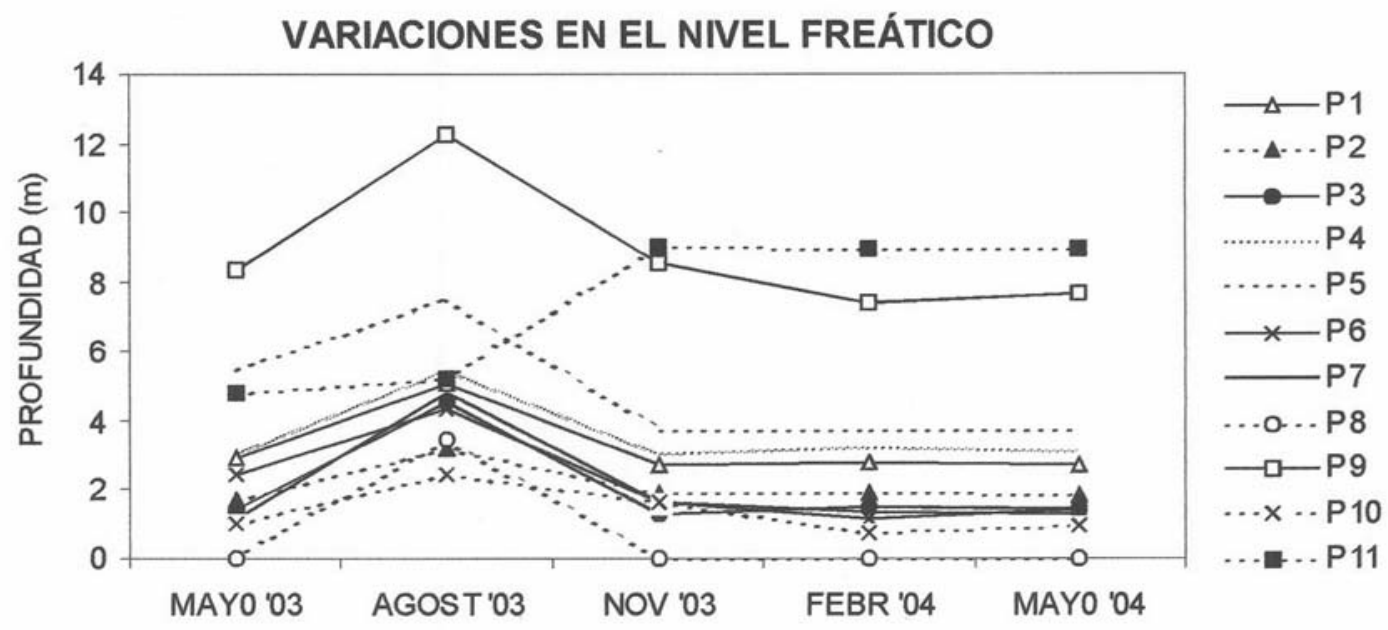

Figura 2. Variaciones estacionales en el nivel freático (respecto a la superficie del suelo). Pozos P1-P11 en el acuífero aluvial del Oja. Seasonal variations in the phreatic level (measured with respect to the ground surface). Wells P1-P11 in the Oja alluvial aquifer.

lo), a fin de conocer las fluctuaciones del nivel piezométrico del acuífero (variaciones naturales: estacionalidad; variaciones artificiales: bombeos, recargas). En todas las estaciones se recogieron muestras de agua: en el río, mediante muestro simple, en los pozos, mediante la creación de muestras integradas a partir de alícuotas recogidas cada dos metros de profundidad. Las profundidades de los pozos oscilaban entre los 4 y 12 $\mathrm{m}$, desde la superficie del nivel freático. Para el muestreo del agua de los pozos se utilizó una botella muestreadora de apertura horizontal de 2.51 . Sobre cada muestra de agua se determinaron las concentraciones de nitrógeno total (NT), nitratos y nitritos. El nitrógeno total se analizó por colorimetría, mediante el método 2.6-dimetil fenol (Lange, 1998). Los nitratos y nitritos se determinaron mediante cromatografía iónica.

El mapa piezométrico de la figura 1, correspondiente a la campaña de mayo de 2003, se creó a partir de los niveles piezométricos de los pozos, trazando las curvas isopiezas por interpolación triangular e interpretativa, con un nivel de precisión del orden de $25 \mathrm{~m}$. Las flechas que indican el sentido del flujo del agua se trazaron desde las líneas de mayor altura piezométrica hacia las líneas de menor altura, en la dirección de la máxima pendiente (perpendiculares a las mismas).

\section{RESULTADOS}

En la figura 1 se representa, en color gris, la Unidad Hidrogeológica del aluvial del Oja, sobre la que se dibuja el mapa piezométrico (correspondiente a mayo de 2003) y el sentido del flujo del agua. El movimiento del agua en el acuífero está condicionado por la topografía. La principal zona de recarga se encuentra entre Ojacastro y Santo Domingo. En la zona media-baja, al reducirse las dimensiones del acuífero, el río Oja y el arroyo Zamaca actúan como drenantes. Las extracciones para riego y abastecimiento, la entrada de agua desde el canal de riego del Najerilla y las intervenciones sobre el cauce del Oja (extracción de áridos, obras de allanamiento del cauce en su curso medio durante 2004, etc.) condicionan el funcionamiento natural del acuífero. La recarga se produce de otoño a primavera, a partir de aguas procedentes de las escorrentías de la Sierra de la Demanda y afloramientos terciarios periféricos, y de la lluvia útil sobre la superficie permeable. El acuífero sufre una importante explotación durante los estiajes. La extracción de agua por bombeo para riego, sumada al menor aporte desde los arroyos tributarios durante el periodo estival, se materializa en un amplio descenso del nivel freático (Fig. 2). El agua llega 


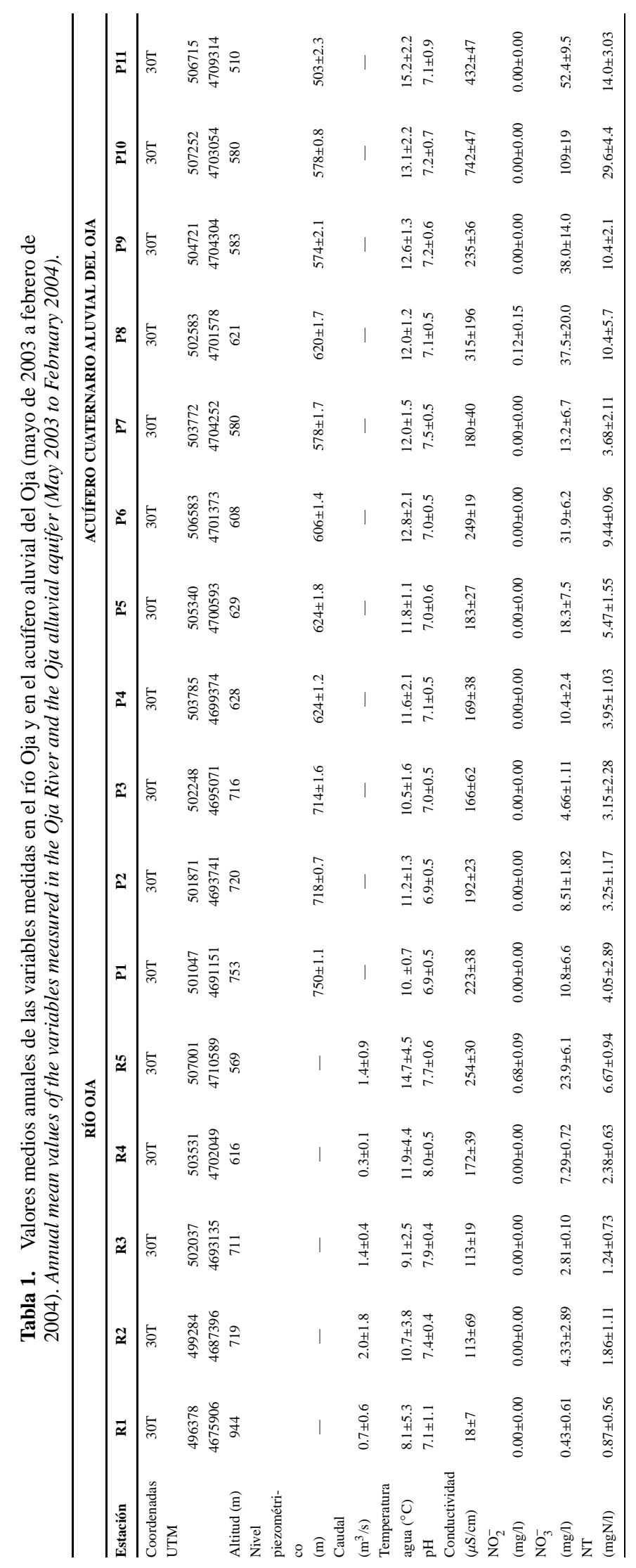



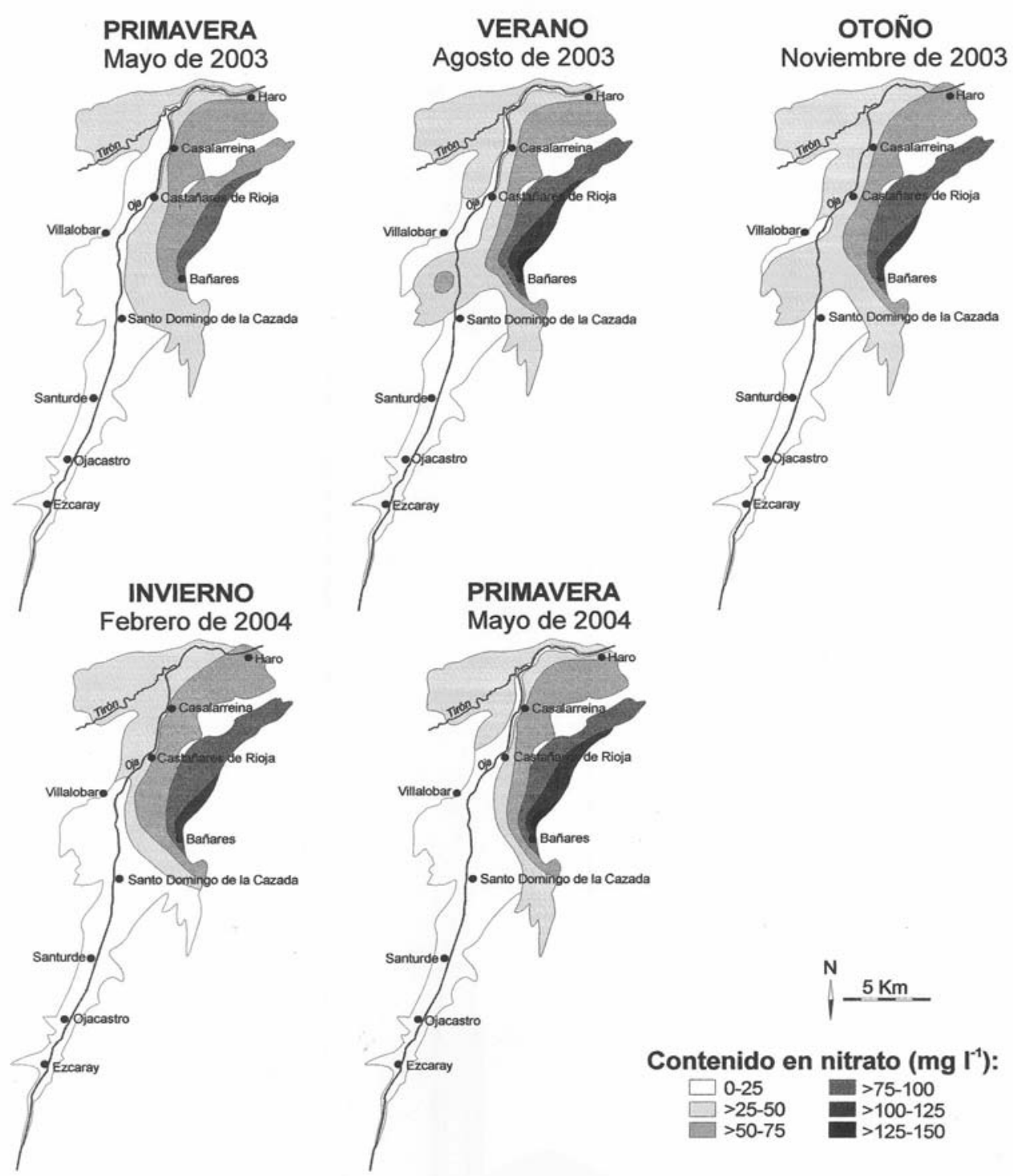

Figura 3. Dinámica espacio-temporal del contenido en nitrato en el acuífero aluvial del Oja. Spatial and temporal dynamics of nitrate content in the Oja alluvial aquifer.

a desaparecer del cauce del Oja, temporalmente, desde Ojacastro hasta Castañares de Rioja. El caudal aumenta de nuevo durante el invierno y el nivel piezométrico se recupera, salvo en la estación P11, en Casalarreina, donde no se observa recuperación en el nivel freático.

En la Tabla 1 se muestran los valores medios anuales de las variables analizadas en cada estación de muestreo, de mayo de 2003 a febrero de 2004. En las zonas sur y centro de la Unidad Hidrogeológica se observa un nivel bajo de mineralización de las aguas, con cierta tendencia a una mineralización moderada hacia el norte, en la zona de descarga del acuífero. En el tercio norte del acuífero las concentraciones de nitrato (Tabla 1, Fig. 3) sobrepasan el límite guía (25 mg/l, Directiva 75/440/CEE) en R5, durante el otoño y el invierno, y en P6, P8, P9, P10 y P11, de forma 
permanente. El límite establecido por la Directiva 91/676/CEE para aguas contaminadas por nitrato $(50 \mathrm{mg} / \mathrm{l})$ se sobrepasa en las estaciones P6, P8, P9, y P11, durante algunos periodos del ciclo anual, mientras que en P10 ocurre a lo largo de todo el año (alcanzándose valores de hasta $128 \mathrm{mg} / \mathrm{l}$ ). Respecto a la aptitud de las aguas para el riego agrícola (Ayers \& Westcot, 1985: FAO $\left.n^{\circ} 29\right)$ se observan restricciones desde ligerasmoderadas hasta severas, debido al exceso de ión nitrato, en los puntos P6, P8, P9, P10 y P11.

En la figura 3 se presenta una secuencia de cinco mapas sobre los que se muestra, a escala estacional, la dinámica espacio-temporal del contenido en nitrato en el acuífero aluvial del Oja. La zona sur del acuífero, que corresponde al área principal de recarga del mismo, conserva siempre un alto nivel de calidad. Se observan, sin embargo, elevadas concentraciones de nitrato en la zona nororiental, zona media-baja del Oja y arroyo Zamaca, donde el acuífero se estrecha. El efecto de la rápida lixiviación del nitrato, procedente de las labores de abonado de la primavera de 2003, se materializa en el mapa de concentración de nitrato de verano de 2003, donde se observa una expansión del área contaminada hacia la zona centro-occidental del acuífero y un agravamiento del proceso de contaminación en la zona este. El carácter cíclico y estacional en el régimen hidrológico, con una alta tasa de recarga del acuífero desde otoño a primavera, a pesar de la sensible sequía y sobreexplotación en verano, permite la recuperación progresiva de la calidad del agua en la zona centro-occidental, efecto ya perceptible en el otoño de 2003 y que continúa hasta la primavera de 2004. Los niveles de calidad en el tramo final del río no se recuperan, sin embargo, hasta la primavera de 2004.

\section{DISCUSIÓN}

Estudios previos efectuados en la cuenca del Oja (Instituto Geológico y Minero de España, 1985) ya señalaban que la fuente de contaminación más importante de la zona eran los abonos nitrogenados de origen agrícola. Se mencionaban entonces concentraciones puntualmente superiores a $40 \mathrm{mg} / \mathrm{l}$, valor tres veces inferior a los máximos observados en la actualidad. Parece evidente, por tanto, el avance progresivo del proceso de contaminación a lo largo de las dos últimas décadas.

Los resultados muestran la capacidad de atenuación natural del proceso de contaminación en la zona centro-occidental del acuífero, como consecuencia de la mayor renovación del agua durante el periodo de otoño a primavera. Los niveles de calidad en el tramo final del río no se recuperan, sin embargo, hasta la primavera de 2004 (Fig. 3). Esta demora parece en concordancia con el hecho de que en la zona entre Castañares y Casalarreina, al reducirse las dimensiones del acuífero, el río Oja actúa como drenante del mismo (Fig. 1). Los valores elevados de nitrato en el tramo final del río durante el otoño y el invierno (Fig. 3) pueden explicarse si se considera que la mayor parte sus aguas proceden del drenaje del área contaminada del acuífero. El proceso difuso de lixiviación de nitrato, intensificado tras las labores de abonado de la primavera anterior, y el posterior transporte del soluto por las aguas subterráneas en el sentido longitudinal del sistema río-acuífero, no sólo afecta a la calidad del propio acuífero sino también, temporalmente, a la calidad de las aguas superficiales.

Las concentraciones de nitrato son elevadas en el área nororiental, zona media-baja del Oja y arroyo Zamaca, donde el acuífero se separa en dos cuencas, Oja y Zamaca, que se estrechan. Aquí, los flujos convergentes y la menor renovación hídrica producen, probablemente, áreas de acumulación. La geología circundante, conformada por conglomerados, areniscas y lutitas, de permeabilidad variable, supone una barrea para el flujo del agua desde las zonas aluviales, altamente permeables, dificultando la capacidad de atenuación natural del proceso de contaminación. La complejidad e irregularidades del sustrato geológico pueden producir discontinuidades temporales o permanentes en las masas de agua subterráneas difíciles de precisar. Aunque se observa, en general, una buena capacidad de recarga del acuífero aluvial, una prueba de estas irregularidades se encuentra en la estación P11 (en Casalarreina), donde el 
nivel freático no se recupera con la llegada del invierno, hecho quizás atribuible a alguna heterogeneidad local en el contexto aluvial.

El estudio de los patrones espaciotemporales de distribución del contenido en nitrato en el sistema río-acuífero, integrando datos químicos e información hidrológica, es el primer paso que debe abordarse para afrontar los problemas derivados de la vulnerabilidad de los acuíferos aluviales a la contaminación por nitrato. Se hace necesario, asimismo, realizar estudios extensivos de campo que permitan interpretar la interacción entre zona no satura y la zona saturada, determinar el papel de los usos del territorio y valorar el efecto de ambos factores sobre la dinámica espaciotemporal del nitrato en las masas de agua. A escala europea, los resultados son todavía tan preliminares que ni siquiera se ha logrado establecer una relación directa entre el aporte de nitrógeno y las mediciones de nitratos en las aguas subterráneas (Nixon et al., 2000), si bien se está trabajando en el desarrollo de modelos para estimar la contribución de las distintas fuentes de nitrógeno y el nitrógeno retenido en el suelo y en las aguas (Grizzetti et al., 2003). Los resultados de este trabajo sugieren que la declaración y seguimiento de un área vulnerable debe plantearse desde un punto de vista dinámico y multidisciplinar, considerando las variaciones espaciotemporales en la totalidad de la Unidad Hidrogeológica e integrando la información química e hidrológica. Por otra parte, la escala temporal de observación empleada para la realización de este estudio (escala estacional) ha resultado satisfactoria, puesto que se encuentra en concordancia tanto con los procesos hidrológicos naturales, como con los usos agrícolas del territorio, proporcionando una amplia información que debe ser completada con el seguimiento de los procesos a largo plazo.

El desarrollo de estudios de estas características proporcionará una base científica sólida para la elaboración y seguimiento de propuestas de regeneración y conservación de las reservas de agua en las zonas vulnerables que permitan compatibilizar unas prácticas agrícolas más optimizadas y una gestión sostenible de los recursos hídricos.

\section{AGRADECIMIENTOS}

Este trabajo de investigación se está financiando a través del Proyecto del Plan Nacional de I+D REN2002-02550. La Unión de Agricultores y Ganaderos-Coordinadora de Agricultores y Ganaderos (UAGR-COAG) apoya el desarrollo de la investigación mediante un Contrato Tecnológico (ref. 2003/672).

Agradecemos muy especialmente a la UAGRCOAG su colaboración y su apoyo logístico en la zona de campo.

La Consejería de Educación de la Comunidad de Madrid y el Fondo Social Europeo (F.S.E.) participan mediante la financiación de una beca de Formación de Personal Investigador.

\section{BIBLIOGRAFÍA}

ARAUZO, M., J.A. DÍEZ \& P. HERNÁIZ 2003. Estimación de balances hídricos y lixiviación de nitratos en sistemas agrícolas. En: Investigación de la zona no saturada, Vol. VI, ZNS'03. J. ÁlvarezBenedí y P. Marinero (eds.): 39-44. Instituto Técnico Agrario de Castilla León, Universidad Europea Miguel de Cervantes. Valladolid, España.

ARCHER, J. \& R. THOMPSON 1993. Solving the nitrate problem: Progress in research and development. AFF Publ., London. 37 pp.

AYERS, R. S. \& D. W. WESTCOT. 1985. Water quality for agriculture. FAO Irrigation and drainage paper, $\mathrm{n}^{\circ}$ 29. Food and Agriculture Organization of the United Nations. Rome. $174 \mathrm{pp}$.

CONFEDERACIÓN HIDROGRÁFICA DEL EBRO 2003. Definición de la red de nitratos en aguas subterráneas, muestreo y determinaciones "in situ". Cuenca del Ebro. Memoria. IPROMA, INOCSA \& ESHYG. Área de Calidad de Aguas, Confederación Hidrográfica del Ebro. 23 pp + Anejo I.

DÍEZ, J.A., R. CABALLERO, R. ROMÁN, A. TARQUIS, M. C. CARTAGENA \& A. VALLEJO 2000. Integrated fertilizer and irrigation management to reduce nitrate leaching in Central Spain. J. Environ. Qual., 29: 1539-1547.

DÍEZ, J. A. 2000. Optimización de la fertilización nitrogenada: procedimiento de análisis de suelo, toma de muestra y elección del tipo de fertilizante. Edafología, 6: 73-84. 
FARES, A. \& K. ALVA 2000. Soil water components based on capacitance probes in a sandy soil. Soil. Sci. Soc. Am. J., 64: 311-318.

GOBIERNO DE LA RIOJA \& ECCYSA 2003. Plan de actuación para reducir la contaminación de las aguas por nitratos agrícolas. Entidad de Control, Certificación, y Servicios Agroalimentarios, Consejería de Turismo y Medio Ambiente de La Rioja, Consejería de Agricultura Ganadería y Desarrollo Rural de La Rioja, Logroño. 11 pp.

GRIZZETTI, B., F. BOURAOUI, G. DE MARSILY \& G. BIDOGLIO 2003. A statistical approach to estimate nitrogen sectorial contribution to total load. Diffuse Pollution Conference, Dublin, Germany: 66-71.

INSTITUTO GEOLÓGICO Y MINERODE ESPAÑA, 1985a. Proyecto de Investigación Hidrogeológica del Cuaternario del río Glera. Comunidad Autónoma de La Rioja. Tomo I: Memoria. Ministerio de Industria y Energía. Zaragoza. 296 pp.

INSTITUTO GEOLÓGICO Y MINERO DE ESPANA,1985b. Plano Hidrogeológico $\mathrm{n}^{\mathrm{o}}$ 1, Investigación Hidrogeológica del Cuaternario del río Glera (La Rioja). Clave: ZP-1490. Escala 1: 50.000 .

INSTITUTO TECNOLÓGICO GEOMINERO DE ESPAÑA. 1998. Mapa de contenido en nitrato de las aguas subterráneas en España, Escala 1:1.500.000. Ministerio de Medio Ambiente, Madrid.

LANGE, 1998. Handbook of photometrical operation analysis. LASA/IP/CADAS/ISIS. Great Britain.

MARTÍNEZ, C. GARCÍA-LINARES, J. M. SÁNCHEZ-PÉREZ, M., A. AIZPURUA, I. RUIZ DE LOIZAGA y I. ANTIGÜEDAD 2003. Lixiviación de nitrato bajo cultivo de remolacha en la zona vulnerable del acuífero cuaternario de Vitoria-Gasteiz (Paçis Vasco). En: Investigación de la zona no saturada, Vol. VI, ZNS'03. J. Álvarez-Benedí y P. Marinero (eds.): 25-32. Instituto Técnico Agrario de Castilla León, Universidad Europea Miguel de Cervantes. Valladolid, España.

MINISTERIO DE MEDIO AMBIENTE 2001.Caracterización de las fuentes agrarias de contaminación de las aguas por nitratos. Secretaría de estado de Aguas y Costas, Dirección General de Obras Hidráulicas y Calidad de Aguas, Madrid. 151 pp. + 11 mapas.

NIXON, S. C., T. J. LACK, D. T. E. HUNT, C. LALLANA \& A. F. BOSCHET 2000. ¿Es sostenible el uso del agua? Situación, perspectivas y pro- blemas. Informe de evaluación ambiental, Agencia Europea de Medio Ambiente. Copenhague. $36 \mathrm{pp}$.

ORTÚZAR, M. A., A. AIZPURUA, A. CASTELLÓN, A. ALONSO y J.M. ESTAVILLO 2003. Evolución del contenido en nitrato y amonio en lixiviados bajo diferentes formas de fertilización nitrogenada en trigo. En: Investigación de la zona no saturada, Vol. VI, ZNS'03. J. ÁlvarezBenedí y P. Marinero (eds.): 39-44. Instituto Técnico Agrario de Castilla León, Universidad Europea Miguel de Cervantes. Valladolid, España.

PALTINEANU, I. C. \& J. L. STARR 1997. Realtime soil water dynamics using multisensor capacitance probes: Laboratory calibration. Soil. Sci. Soc. Am. J., 61: 1576-1585.

RASS, D.P., J. T. RITCHIE, W. R. PETERSON, T. L. LOUDON \& E. C. MARTIN 1999. Nitrogen management impacts on yield and nitrate leaching in inbred maize systems. J. Environ. Qual., 28: 1365-1371.

RAMOS, C. \& M. KÜCKE 1999. Revisión crítica de los métodos de medida de la lixiviación de nitrato en suelos agrícolas. En: Estudios de la zona no saturada del suelo. R. Muñoz-Carpena, A. Ritter, C. Tascón (eds.): 25-32. ICIA. Tenerife.

ROMÁN, R., R. CABALLERO, A. BUSTOS, J. A. DÍEZ, M. C. CARTAGENA, A. VALLEJO \& A. CABALLERO 1996. Water and solute movement under conventional corn in Central Spain: I. Water balance. Soil. Sci. Soc. Am. J., 60: 1530-1536.

SÁNCHEZ, L., J. A. DÍEZ, A. VALLEJO, M. C. CARTAGENA \& A. POLO 1998. Estimate of mineralized organic nitrogen in soil using nitrogen balances and determining available nitrogen by the electro-ultrafiltration technique. Application to Mediterranean climate soil. J. Agric. Food Chem., 46: 2036-2043.

STARR, J. L. \& I. C. PALTINEANU 1998. Soil water dynamics using multisensor capacitance probes in nontraffic interrrows of corn. Soil Sci. Soc. Am. J., 62: 114-122.

VÁZQUEZ, N., A. PARDO, M. L. SUSO y M. QUEMADA 2003. Drenaje y lixiviación de nitratos en una sección transversal de un sistema de cultivo hortícola con riego por goteo y acolchado plástico. En: Investigación de la zona no saturada, Vol. VI, ZNS'03. J. Álvarez-Benedí y P. Marinero (eds.): 33-38. Instituto Técnico Agrario de Castilla León, Universidad Europea Miguel de Cervantes. Valladolid, España. 